\title{
Characterization of Bushy Cashew (Anacardium humile A. St.-Hil.) in the State of Goiás, Brazil
}

\author{
Laísse D. Pereira ${ }^{1}$, Danielle F. P. Silva ${ }^{1}$, Edésio F. Reis ${ }^{1}$, Jefferson F. N. Pinto ${ }^{1}$, Hildeu F. Assunção ${ }^{1}$, \\ Carla G. Machado ${ }^{1}$, Francielly R. Gomes ${ }^{1}$, Luciana C. Carneiro ${ }^{1}$, Simério C. S. Cruz ${ }^{1}$ \& Claudio H. M. Costa ${ }^{1}$ \\ ${ }^{1}$ Regional Jataí, Federal University of Goiás, Goiás, Brazil \\ Correspondence: Laísse D. Pereira, Regional Jataí, Federal University of Goiás, Cidade Universitária José \\ Cruciano de Araújo (Jatobá), Rod BR 364 KM 192-Parque Industrial, 3800, CEP 75801-615, Jataí, Goiás, Brazil. \\ E-mail: laissedaniellep@gmail.com
}

Received: January 1, 2019

doi:10.5539/jas.v11n5p183

\author{
Accepted: February 4, 2019 Online Published: April 15, 2019 \\ URL: https://doi.org/10.5539/jas.v11n5p183
}

\begin{abstract}
The physical and chemical characteristics of the fruits influence the consumer acceptance. The objective of this study was to perform the physical, physico-chemical and chemical characterization of fruits of accessions of bushy cashew (Anacardium humile A. St.-Hil.) (cashew nut and cashew apple) in a germplasm bank located in southwest of the state of Goiás, in Brazil, aiming at the selection of superior accessions, in order to facilitate the initiation of a program to encourage the production and consumption, for provide information for breeding programs and the specie preservation. This research study was conducted at the Laboratory of genetics and molecular biology with material collected in the Experimental Station of the Federal University of Jataí, within the biological ex situ collection of Anacardium humile, in the field of genetic resources. In the harvest of 2016, within this collection, they were evaluated accesses for the characteristics of the cashew apple: length, diameter of base, apex diameter, weight, instrumental coloration of the epidermis and pulp, titratable acidity (citric acid), vitamin $\mathrm{C}$ content (ascorbic acid) and carotenoid content; cashew nut were evaluated: length, width, thickness and weight. The results were submitted to descriptive analysis, obtaining average, minimum, maximum and variation coefficient and the correlation between the variables, testing the significance by the $t$ test. Most of the accesses showed high CV for most of the variables, demonstrating high heterogeneity of the observed values. Cashew apples have high levels of vitamin $\mathrm{C}$ and carotenoids, indicating the nutritional potential of the specie.
\end{abstract}

Keywords: cerrado biome, native fruit, nutritional quality

\section{Introduction}

The native fruit crops occupy a prominent place in the Cerrado biome and its fruits have great popular acceptance (Avidos \& Ferreira, 2000). Among them, stands the species Anacardium humile A. St.-Hil., which is distributed in the different States in which the biome occupies, its fruits are popularly known as 'cajuzinho-do-campo', 'cajuzinho-do-cerrado' (bushy cashew) or 'cajuí' (Silva-Luz \& Pirani, 2010) and it is divided into two parts: the fruit itself, which is popularly known as cashew nut, and cashew apple, technically called floral peduncle, which it is the part sold as fruit (Lima, 1988).

As bushy cashew is recognized as a nutritional reference of great importance for local population, such species became a fruit of high potential for sustainable exploitation of the Cerrado biome, turnning its inclusion in the "crops for large-scale production" an important action, avoiding predatory exploitation and extinction risk (Londe, Ribeiro, Sousa, Kerr, \& Bonetti, 2010). Morphological studies of fruits, seeds and chemical characterization of the pulp are frequent for several species. These are generally performed for pre-breeding programs of not domesticated species (Moura, Chaves, \& Naves, 2013) and such studies detect the genetic variability between individuals or accesses in a population (Almeida Junior, Chaves, \& Soares, 2014).

The description of bushy cashew's fruits characteristics are important to give technical support to its commercial exploitation. According to Silva et al. (2012), the characterization allows the study of genetic diversity among accessions or populations and the identification of potential parents, or even genotypes with superior characteristics. Information regarding these characteristics and nutritional value of fruits are basic tools for assessment of consumption and the formulation of new products (Silva, Lacerda, Santos, \& Martins, 2008). 
Early estimates of existing variability are obtained from the characterization of the genetic material, being one of the steps to access genetic diversity, both within and among plant accesses, since observations and measurements of several phenotypic descriptors are easily detectable (Burle \& Oliveira, 2010). These results are then used in conjunction with other characterization techniques, such as DNA markers, cytogenetic techniques, among others (Bruno et al., 2017).

The Characterization of native fruit becomes essential to differentiate genotypes in germplasm banks and also as an auxiliary tool in breeding programs. Therefore, the objective of this study was to perform the physical, physico-chemical and chemical characterization of fruits of accessions of bushy cashew in a germplasm bank located in southwest of the state of Goiás, in Brazil, aiming at the selection of superior accessions, in order to facilitate the initiation of a program to encourage the production and consumption, for provide information for breeding programs and the specie preservation.

\section{Materials and Methods}

\subsection{Area Description}

This study was carried out at the genetics and molecular biology laboratory with the material collected from the biological ex situ collection of Anacardium humile, located at the genetic resources site at the Experimental Station of the Federal University of Jataí (17 $53^{\prime} 08^{\prime \prime} \mathrm{N}$ and $\left.51^{\circ} 40^{\prime} 12^{\prime \prime} \mathrm{W}\right)$.

The area has a tropical savanna climate classification (Aw), with the rainy period from October to April and dry from May to September. The average annual temperature varies between $21^{\circ} \mathrm{C}$ and $23{ }^{\circ} \mathrm{C}$ and the average annual rainfall of $1700 \mathrm{~mm}$.

The collection contains 542 plants (accessions). The seeds sampling for structuring the collection was carried out in October 2011 and 2012 in 14 counties of the state of Goiás (Brazil), and the planting of seedlings was carried out in December 2011 and March 2013, which occupies an area of approximately $1.720 \mathrm{~m}^{2}$.

\subsection{Material Studied}

For each access it was collected from 5 to 15 units of bushy cashew nuts and apples. One of the fruits collect criteria was the cashew apple epidermis color, that should be fully yellow coloration or fully red, that is, cashew apple fully ripe.

Most of the accessions did not bear fruit, so they were collected from accessions generated through material coming from only seven (Caiapônia, Chapadão do Céu, Itarumã, Jataí, Mineiros, Portelândia and Serranópolis) out of the 14 counties, which were enumerated in order to facilitate the understanding of the results (Table 1). 
Table 1. Characterization of the accessions of bushy cashew evaluated

\begin{tabular}{|c|c|c|}
\hline Accessions & Provenance & Number of cashew nut/cashew apple \\
\hline 1 & Caiapônia & 13 \\
\hline 2 & Serranópolis & 15 \\
\hline 3 & Serranópolis & 15 \\
\hline 4 & Serranópolis & 15 \\
\hline 5 & Serranópolis & 8 \\
\hline 6 & Jataí & 14 \\
\hline 7 & Chapadão do céu & 15 \\
\hline 8 & Chapadão do céu & 10 \\
\hline 9 & Chapadão do céu & 5 \\
\hline 10 & Mineiros & 15 \\
\hline 11 & Mineiros & 12 \\
\hline 12 & Itarumã & 11 \\
\hline 13 & Itarumã & 8 \\
\hline 14 & Itarumã & 15 \\
\hline 15 & Itarumã & 13 \\
\hline 16 & Itarumã & 5 \\
\hline 17 & Itarumã & 13 \\
\hline 18 & Jataí & 6 \\
\hline 19 & Portelândia & 12 \\
\hline 20 & Portelândia & 5 \\
\hline 21 & Serranópolis & 5 \\
\hline 22 & Serranópolis & 14 \\
\hline 23 & Serranópolis & 13 \\
\hline 24 & Mineiros & 13 \\
\hline 25 & Mineiros & 5 \\
\hline 26 & Mineiros & 7 \\
\hline 27 & Mineiros & 15 \\
\hline
\end{tabular}

\subsection{Data Collection}

The cashew apple weight (WEA) and cashew nut weight (WEN) were obtained using a digital scale, being expressed in grams. The cashew apple length (LEA), cashew apple base diameter (BDA), cashew apple apex diameter (DAA), cashew nut length (LEN), cashew nut width (WIN) and cashew nut thickness (THN) were measured in millimeters with the aid of a digital caliper.

The epidermis color (measured in the equatorial region of one of the faces of the cashew apple) and the pulp was amended by coordinated $\mathrm{C}^{*}$ and Hue angle $\left(\mathrm{h}^{\circ}\right)$, determined with the aid of Konica Minolta colorimeter, Model CR-10, which measures the reflected light, using a cartesian coordinate system $\mathrm{L}^{*}, \mathrm{a}^{*}$ and $\mathrm{b}^{*}$, which it gets points or units of approximate visual uniformity. The value $a^{*}$ corresponds to red-green scale and varies from +60 to -60 , corresponding negative values to the different shades of green and the positives, to red shades. The parameter $b^{*}$ corresponds to scale yellow-blue and also varies from +60 to -60 , emphasizing that the negative values correspond to shades of blue and the positives, the shades of color yellow. The $\mathrm{L}^{*}$ refers to the level of brightness, ranging from 0 (black color) to 100 (white color).

The content of soluble solids (SSA) of cashew apple were analyzed by using a portable refractometer, expressed in ${ }^{\circ}$ Brix; acidity (content of citric acid) (CAA), taking the titrations, under agitation, with $0.1 \mathrm{~N} \mathrm{NaOH}$ solution, using the phenolphthalein solution $1 \%$ as an indicator; soluble solids and acidity ratio (ratio) was obtained by dividing the values of soluble solids by titratable acidity content; vitamin C (content of ascorbic acid) (VITC) determined by titration with the Folin-Tillman [2.6 diclorofenolindofenol (sodium salt) $0.1 \%$ ] (Instituto Adolfo Lutz, 2008) and that carotenoids (CRT) were extracted with acetone and analyzed in a spectrophotometer, the absorbences were determined at 470,646.8 and $663.2 \mathrm{~nm}$ and carotenoid levels determined in accordance with the equations of Lichtenthaler (1987). 


\subsection{Data Analysis}

The obtained data were submitted to descriptive statistical analyzes, on the average, minimum, maximum, coefficient of variation and the correlation between the variables, testing the significance by the test with the GENES software (Cruz, 2013).

\section{Results}

\subsection{Physical Traits of Cashew Apples}

The mean values for the physical characteristics of cashew apple are presented in Table 2, where LEA ranged from 16.50 to $37.92 \mathrm{~mm}$ among accessions, and WEA values were found between 2.18 and $18.78 \mathrm{~g}$. For diameter, basal and apex values of cashew apple, BDA mean values ranged from 9.42 to $20.80 \mathrm{~mm}$ and DAA from 17.58 to $30.61 \mathrm{~mm}$. There was no access that stood out in both mentioned characteristics.

Table 2. Average (Ave), minimum (Min) and maximum (Max) values; and coefficient of variation (CV) referring to the physical characteristics of cashew apple of bushy cashew access

\begin{tabular}{|c|c|c|c|c|c|c|c|c|c|c|c|c|c|c|c|c|}
\hline \multirow{2}{*}{ Access } & \multicolumn{4}{|c|}{ LEA } & \multicolumn{4}{|c|}{ BDA } & \multicolumn{4}{|c|}{ DAA } & \multicolumn{4}{|c|}{ WEA } \\
\hline & Min & Ave & Max & $\mathrm{CV}$ & Min & Ave & Max & $\mathrm{CV}$ & Min & Ave. & Max & $\mathrm{CV}$ & Min & Ave & Max & $\mathrm{CV}$ \\
\hline 1 & 23.09 & 26.62 & 30.20 & 8.13 & 11.46 & 14.73 & 17.37 & 13.91 & 18.87 & 23.59 & 28.53 & 11.64 & 4.47 & 7.03 & 10.99 & 26.90 \\
\hline 2 & 19.88 & 24.47 & 30.35 & 12.12 & 9.11 & 16.37 & 22.16 & 24.19 & 19.07 & 25.20 & 30.73 & 12.42 & 2.92 & 7.16 & 16.13 & 45.57 \\
\hline 3 & 21.00 & 24.46 & 27.45 & 6.81 & 9.47 & 13.23 & 22.47 & 30.45 & 16.60 & 23.60 & 27.97 & 10.58 & 2.99 & 6.42 & 9.54 & 23.91 \\
\hline 4 & 22.53 & 29.49 & 35.46 & 11.70 & 8.02 & 12.68 & 17.85 & 21.62 & 18.62 & 25.19 & 29.35 & 10.46 & 4.71 & 8.45 & 12.74 & 23.89 \\
\hline 5 & 34.05 & 36.45 & 39.38 & 4.51 & 14.48 & 21.10 & 26.19 & 20.90 & 23.52 & 33.95 & 37.33 & 12.98 & 7.43 & 18.78 & 24.64 & 27.58 \\
\hline 6 & 20.96 & 23.87 & 31.66 & 12.39 & 13.81 & 16.65 & 18.97 & 9.36 & 24.19 & 26.02 & 28.82 & 5.41 & 5.47 & 7.27 & 9.78 & 16.37 \\
\hline 7 & 14.88 & 18.44 & 21.05 & 9.43 & 7.00 & 11.11 & 15.03 & 17.65 & 16.97 & 20.03 & 23.37 & 8.74 & 1.99 & 3.63 & 5.14 & 21.99 \\
\hline 8 & 16.41 & 19.93 & 23.95 & 11.21 & 11.66 & 13.65 & 15.66 & 9.92 & 19.36 & 22.21 & 24.92 & 9.18 & 2.91 & 4.65 & 6.69 & 28.04 \\
\hline 9 & 19.79 & 23.85 & 27.23 & 12.52 & 12.92 & 17.63 & 23.55 & 21.76 & 25.38 & 28.68 & 32.79 & 10.67 & 0.23 & 7.76 & 16.68 & 77.16 \\
\hline 10 & 15.83 & 22.33 & 29.47 & 16.58 & 12.94 & 16.52 & 20.61 & 14.26 & 20.00 & 25.19 & 30.08 & 10.87 & 3.22 & 6.71 & 12.60 & 39.00 \\
\hline 11 & 12.61 & 17.36 & 22.78 & 17.95 & 5.91 & 13.55 & 22.75 & 35.83 & 14.07 & 20.00 & 26.74 & 17.67 & 1.15 & 3.92 & 8.09 & 52.83 \\
\hline 12 & 19.73 & 22.34 & 31.00 & 14.34 & 9.82 & 14.51 & 18.11 & 21.31 & 15.73 & 20.71 & 26.11 & 14.26 & 2.47 & 5.08 & 10.67 & 44.32 \\
\hline 13 & 13.57 & 16.50 & 19.51 & 10.53 & 7.48 & 9.42 & 13.70 & 21.10 & 14.46 & 17.58 & 20.77 & 11.41 & 1.12 & 2.18 & 2.93 & 28.72 \\
\hline 14 & 14.51 & 19.59 & 31.90 & 27.17 & 7.07 & 12.67 & 21.64 & 32.47 & 9.41 & 18.45 & 31.43 & 28.01 & 1.16 & 4.08 & 15.05 & 87.18 \\
\hline 15 & 23.17 & 27.64 & 34.15 & & & 12.50 & 17.54 & 30.73 & 19.06 & 23.50 & 28.19 & & 3.79 & 7.00 & 13.57 & 40.24 \\
\hline 16 & 20.38 & 23.19 & 29.65 & 16.18 & 11.41 & 16.92 & 19.31 & & 24.73 & 28.69 & 33.24 & 11.03 & 5.36 & 9.24 & 15.20 & 39.63 \\
\hline 17 & 16.00 & 24.72 & 30.89 & 14.43 & 12.42 & 15.11 & 17.71 & 12.12 & 21.80 & 25.19 & 28.02 & 6.74 & 4.60 & 7.66 & 10.86 & 24.12 \\
\hline 18 & 15.36 & 17.14 & 20.01 & 10.21 & 10.07 & 11.01 & 11.71 & 6.22 & 16.31 & 19.83 & 21.88 & 9.69 & 2.48 & 3.61 & 4.66 & 19.90 \\
\hline 19 & 18.35 & 23.32 & 27.53 & 10.97 & 9.43 & 17.59 & 21.34 & 16.22 & 20.45 & 25.82 & 28.60 & 8.53 & 4.52 & 8.35 & 10.65 & 21.04 \\
\hline 20 & 30.19 & 37.92 & 43.93 & 14.36 & 12.05 & 15.59 & 17.20 & 12.99 & 19.85 & 25.89 & 28.63 & 14.05 & 5.80 & 11.89 & 15.67 & 34.47 \\
\hline 21 & 17.32 & 37.83 & 49.87 & 40.09 & 9.55 & 16.16 & 20.26 & 24.60 & 13.87 & 24.00 & 28.90 & 26.38 & 2.16 & 13.15 & 20.40 & 50.93 \\
\hline 22 & 16.11 & 21.17 & 32.94 & 21.19 & 11.49 & 17.77 & 31.22 & 28.86 & 16.19 & 21.81 & 27.07 & 13.99 & 3.16 & 6.07 & 9.14 & 32.74 \\
\hline 23 & 22.00 & 26.77 & 29.80 & 10.19 & 9.94 & 20.80 & 25.08 & 20.04 & 26.04 & 31.82 & 35.69 & 10.08 & 2.44 & 12.16 & 19.32 & 35.48 \\
\hline 24 & 12.39 & 16.78 & 18.83 & 10.46 & 12.35 & 15.77 & 19.02 & 11.82 & 19.01 & 23.15 & 29.10 & 11.71 & 1.02 & 3.96 & 6.05 & 32.43 \\
\hline 25 & 12.99 & 22.75 & 32.57 & 30.65 & 9.17 & 14.75 & 19.47 & 28.06 & 19.70 & 22.27 & 25.07 & 9.29 & 3.26 & 4.70 & 6.94 & 33.04 \\
\hline 26 & 29.02 & 32.04 & 34.94 & 6.85 & 13.19 & 18.51 & 24.37 & 23.60 & 28.20 & 30.61 & 33.48 & 6.82 & 8.72 & 13.56 & 18.51 & 25.80 \\
\hline 27 & 11.90 & 22.69 & 25.97 & 15.01 & 11.69 & 14.32 & 16.98 & 8.38 & 19.40 & 24.33 & 26.94 & 8.96 & 3.04 & 6.56 & 8.70 & 23.09 \\
\hline
\end{tabular}

Note. LEA: length of the cashew apple (mm), BDA: base diameter of cashew apple (mm), DAA: diameter of the apex of cashew apple ( $\mathrm{mm})$ and WEA: weight of cashew apple $(\mathrm{g})$.

\subsection{Cashew Apple's color Attributes}

According to Table 3, access 1 presented lower values of epidermis hue angle (hEA), which indicates higher values of "a" in relation to "b" and, consequently, epidermis with red coloration. The remainig accessions presented mean values close to each other and higher values of "b" in relation to "a", indicating that these accesses presented yellowish coloration. 
Table 3. Average (Ave), minimum (Min) and maximum (Max) values; and coefficient of variation (CV) referring to the coloration characteristics of cashew apple of bushy cashew access

\begin{tabular}{|c|c|c|c|c|c|c|c|c|c|c|c|c|c|c|c|c|}
\hline \multirow{2}{*}{ Access } & \multicolumn{4}{|c|}{ CEA } & \multicolumn{4}{|c|}{ hEA } & \multicolumn{4}{|c|}{$\mathrm{CPA}$} & \multicolumn{4}{|c|}{$\mathrm{hPA}$} \\
\hline & Min & Ave & Max & $\mathrm{CV}$ & Min & Ave & Max & $\mathrm{CV}$ & Min & Ave & Max & $\mathrm{CV}$ & Min & Ave & Max & $\mathrm{CV}$ \\
\hline 1 & 39.40 & 40.70 & 43.10 & 5.11 & 9.40 & 9.70 & 10.00 & 3.09 & 6.70 & 10.37 & 15.30 & 42.81 & 32.70 & 49.63 & 73.30 & 42.55 \\
\hline 2 & 32.30 & 34.83 & 37.20 & 7.05 & 83.30 & 85.73 & 88.90 & 3.35 & 21.20 & 24.67 & 30.90 & 21.93 & 95.30 & 100.93 & 105.10 & 5.01 \\
\hline 3 & 42.90 & 49.20 & 56.90 & 14.44 & 83.30 & 86.20 & 89.80 & 3.83 & 37.30 & 40.67 & 45.50 & 10.55 & 93.70 & 97.00 & 99.60 & 3.10 \\
\hline 4 & 35.90 & 36.67 & 37.60 & 2.35 & 82.00 & 85.83 & 88.50 & 3.96 & 19.80 & 22.03 & 23.20 & 8.78 & 98.50 & 104.10 & 109.30 & 5.20 \\
\hline 5 & 37.60 & 40.80 & 46.80 & 12.74 & 81.50 & 82.77 & 84.90 & 2.24 & 11.20 & 12.80 & 14.00 & 11.27 & 110.50 & 114.03 & 116.60 & 2.77 \\
\hline 6 & 38.80 & 43.37 & 46.30 & 9.24 & 82.60 & 84.37 & 85.30 & 1.81 & 32.20 & 33.57 & 35.00 & 4.17 & 95.00 & 97.23 & 98.50 & 1.99 \\
\hline 7 & 30.40 & 33.03 & 37.60 & 12.02 & 38.30 & 54.30 & 67.80 & 27.45 & 19.40 & 20.87 & 22.30 & 6.95 & 104.60 & 104.80 & 105.10 & 0.25 \\
\hline 8 & 40.20 & 44.17 & 46.80 & 7.97 & 75.70 & 83.07 & 88.20 & 7.88 & 42.30 & 43.00 & 43.70 & 1.63 & 91.40 & 95.50 & 97.80 & 3.73 \\
\hline 9 & 32.80 & 34.47 & 36.80 & 6.04 & 54.20 & 69.30 & 89.60 & 26.35 & 22.40 & 24.73 & 26.30 & 8.33 & 101.90 & 103.17 & 104.40 & 1.21 \\
\hline 10 & 41.80 & 43.73 & 46.20 & 5.14 & 89.30 & 90.80 & 92.10 & 1.55 & 21.70 & 23.10 & 25.00 & 7.38 & 101.80 & 104.57 & 106.20 & 2.30 \\
\hline 11 & 38.70 & 41.03 & 42.30 & 4.93 & 89.20 & 91.00 & 93.30 & 2.30 & 34.30 & 39.37 & 46.70 & 16.52 & 91.40 & 94.27 & 96.60 & 2.80 \\
\hline 12 & 40.40 & 43.63 & 46.10 & 6.71 & 85.10 & 92.50 & 97.10 & 7.00 & 31.10 & 31.87 & 33.00 & 3.14 & 98.30 & 98.90 & 100.00 & 0.96 \\
\hline 13 & 28.10 & 30.33 & 32.80 & 7.78 & 49.20 & 53.67 & 60.50 & 11.20 & 27.60 & 33.37 & 39.20 & 17.38 & 83.70 & 91.83 & 97.40 & 7.84 \\
\hline 14 & 29.00 & 35.20 & 40.30 & 16.28 & 87.70 & 90.93 & 96.50 & 5.32 & 21.50 & 24.83 & 28.70 & 14.61 & 102.50 & 104.97 & 107.00 & 2.17 \\
\hline 15 & 39.40 & 40.25 & 41.10 & 2.99 & 94.30 & 94.50 & 94.70 & 0.29 & 26.50 & 29.70 & 32.90 & 15.24 & 96.60 & 97.70 & 98.80 & 1.59 \\
\hline 16 & 20.10 & 32.30 & 43.90 & 36.88 & 41.80 & 69.20 & 89.30 & 35.52 & 23.30 & 30.10 & 36.00 & 21.25 & 95.80 & 98.63 & 100.90 & 2.63 \\
\hline 17 & 44.30 & 45.63 & 46.70 & 2.68 & 77.80 & 81.77 & 85.90 & 4.96 & 29.00 & 30.73 & 32.10 & 5.15 & 93.80 & 95.50 & 98.30 & 2.56 \\
\hline 18 & 47.10 & 49.23 & 52.00 & 5.10 & 88.72 & 89.37 & 90.00 & 0.72 & 29.60 & 29.83 & 30.20 & 1.08 & 98.70 & 99.27 & 100.00 & 0.67 \\
\hline 19 & 42.60 & 44.10 & 44.90 & 2.95 & 88.70 & 90.63 & 93.80 & 3.05 & 25.30 & 28.47 & 32.80 & 13.64 & 99.20 & 101.37 & 102.80 & 1.88 \\
\hline 20 & 34.40 & 39.73 & 48.00 & 18.27 & 85.50 & 89.47 & 91.60 & 3.84 & 21.80 & 23.50 & 24.90 & 6.69 & 100.50 & 102.40 & 104.60 & 2.02 \\
\hline 21 & 34.50 & 40.13 & 44.90 & 13.09 & 82.40 & 88.30 & 94.70 & 6.98 & 20.80 & 22.00 & 22.70 & 4.75 & 102.40 & 103.07 & 103.70 & 0.63 \\
\hline 22 & 32.90 & 44.00 & 53.40 & 23.53 & 86.70 & 93.67 & 99.50 & 6.91 & 24.00 & 30.03 & 35.70 & 19.51 & 97.40 & 100.10 & 103.60 & 3.17 \\
\hline 23 & 41.00 & 44.60 & 48.00 & 7.86 & 85.00 & 86.57 & 88.30 & 1.91 & 22.80 & 24.00 & 24.80 & 4.41 & 98.70 & 101.37 & 104.60 & 2.95 \\
\hline 24 & 39.50 & 42.87 & 46.80 & 8.59 & 77.40 & 84.80 & 90.90 & 8.07 & 28.60 & 32.30 & 35.10 & 10.35 & 95.00 & 98.20 & 100.90 & 3.04 \\
\hline 25 & 36.00 & 37.60 & 38.80 & 3.84 & 98.20 & 101.07 & 103.60 & 2.69 & 19.40 & 25.33 & 29.70 & 21.02 & 101.80 & 105.00 & 107.70 & 2.84 \\
\hline 26 & 31.20 & 36.80 & 41.70 & 14.36 & 88.60 & 94.20 & 98.10 & 5.28 & 28.30 & 29.77 & 32.00 & 6.60 & 97.60 & 98.03 & 98.70 & 0.60 \\
\hline 27 & 26.20 & 30.57 & 33.90 & 12.93 & 88.30 & 91.90 & 94.20 & 3.44 & 23.00 & 26.70 & 30.10 & 13.33 & 100.20 & 102.47 & 103.90 & 1.94 \\
\hline
\end{tabular}

Note. CEA: coordinated $\mathrm{C}^{*}$ of cashew apple epidermis, hEA: hue angle $\left(\mathrm{h}^{\circ}\right)$ of cashew apple epidermis, CPA: coordinated $\mathrm{C}^{*}$ of cashew apple pulp and hPA: hue angle $\left(\mathrm{h}^{\circ}\right)$ of cashew apple pulp.

\subsection{Chemical and Physico-chemical Traits of Cashew Apple}

The chemical/nutritional information of cashew apple is shown in Tables 4 and 5 . The mean values of SSA showed a variation between the accessions from 5.29 to $12.40^{\circ}$ Brix (Table 4). High SSA values are interesting both for in natura consumption and for processing industry. The variation observed for CAA percentage was from 0.66 to $1.65 \%$. The average values found for ratio ranged from 3.88 to 13.78 , showing great variation in flavor intensity within species. The CRT content varied from 0.10 to $0.48 \%$ in average values of accessions species (Table 5). For the characteristic VITC there was variation between the average values of 74.09 to 279.65 $\mathrm{mg} / 100 \mathrm{~g}$ of pulp, being the accessions 1 and 3 with higher values. 
Table 4. Average (Ave), minimum (Min) and maximum (Max) values; and coefficient of variation (CV) referring to the chemical characteristics of cashew apple of bushy cashew access

\begin{tabular}{|c|c|c|c|c|c|c|c|c|c|c|c|c|}
\hline \multirow{2}{*}{ Access } & \multicolumn{4}{|c|}{ SSA } & \multicolumn{4}{|c|}{ CAA } & \multicolumn{4}{|c|}{ ratio } \\
\hline & Min & Ave & Max & $\mathrm{CV}$ & Min & Ave & Max & $\mathrm{CV}$ & Min & Ave & Max & $\mathrm{CV}$ \\
\hline 1 & 9.56 & 11.27 & 14.00 & 21.16 & 1.25 & 1.36 & 1.56 & 12.78 & 6.57 & 8.41 & 10.99 & 27.36 \\
\hline 2 & 7.76 & 07.99 & 08.10 & 02.46 & 1.05 & 1.08 & 1.11 & 2.79 & 7.00 & 7.38 & 7.72 & 04.87 \\
\hline 3 & 9.48 & 12.40 & 17.38 & 34.95 & 0.95 & 1.14 & 1.32 & 22.96 & 9.94 & 11.54 & 13.14 & 19.57 \\
\hline 4 & 8.02 & 10.03 & 11.46 & 17.85 & 1.21 & 1.25 & 1.32 & 4.79 & 6.62 & 8.01 & 9.38 & 17.24 \\
\hline 5 & 4.00 & 05.55 & 07.30 & 29.86 & 1.11 & 1.14 & 1.20 & 4.05 & 3.60 & 4.86 & 6.51 & 30.74 \\
\hline 6 & 4.08 & 05.40 & 06.30 & 21.63 & 1.08 & 1.11 & 1.13 & 2.30 & 3.63 & 4.86 & 5.81 & 22.89 \\
\hline 7 & 7.92 & 09.05 & 10.14 & 12.27 & 1.34 & 1.41 & 1.46 & 4.36 & 5.59 & 6.43 & 6.94 & 11.45 \\
\hline 8 & 10.34 & 11.26 & 12.50 & 09.90 & 0.77 & 0.82 & 0.85 & 5.46 & 12.88 & 13.78 & 14.96 & 07.75 \\
\hline 9 & 7.00 & 09.40 & 12.00 & 26.66 & 1.22 & 1.26 & 1.32 & 4.34 & 5.31 & 7.54 & 9.85 & 30.10 \\
\hline 10 & 6.20 & 07.26 & 08.20 & 13.85 & 0.79 & 0.81 & 0.82 & 1.63 & 7.56 & 9.00 & 10.15 & 14.68 \\
\hline 11 & 4.90 & 05.29 & 06.06 & 12.67 & 0.93 & 0.97 & 1.02 & 5.00 & 4.80 & 5.49 & 6.52 & 16.58 \\
\hline 12 & 4.80 & 08.90 & 15.00 & 60.52 & 1.11 & 1.12 & 1.15 & 1.93 & 4.30 & 7.95 & 13.55 & 61.91 \\
\hline 14 & 7.60 & 08.57 & 10.06 & 15.25 & 1.25 & 1.31 & 1.35 & 4.29 & 5.67 & 6.53 & 7.45 & 13.70 \\
\hline 15 & 9.68 & 11.89 & 14.72 & 21.67 & 1.01 & 1.05 & 1.07 & 3.22 & 9.06 & 11.33 & 13.77 & 20.84 \\
\hline 16 & 5.00 & 07.00 & 09.40 & 31.82 & 1.15 & 1.17 & 1.20 & 2.31 & 4.34 & 5.96 & 8.05 & 31.82 \\
\hline 17 & 10.20 & 10.86 & 11.70 & 07.07 & 1.10 & 1.14 & 1.19 & 3.72 & 8.98 & 9.51 & 10.31 & 07.44 \\
\hline 19 & 9.32 & 11.03 & 13.40 & 19.23 & 1.57 & 1.58 & 1.61 & 1.31 & 5.93 & 6.96 & 8.34 & 17.89 \\
\hline 20 & 6.80 & 07.40 & 08.00 & 08.11 & 0.62 & 0.66 & 0.71 & 7.50 & 10.60 & 11.27 & 11.98 & 06.15 \\
\hline 21 & 6.00 & 07.67 & 10.00 & 27.15 & 0.93 & 0.96 & 0.98 & 2.38 & 6.27 & 8.05 & 10.73 & 29.31 \\
\hline 22 & 9.30 & 10.21 & 10.70 & 07.71 & 1.16 & 1.17 & 1.19 & 1.22 & 8.03 & 8.71 & 9.08 & 06.76 \\
\hline 23 & 5.17 & 06.40 & 08.00 & 22.73 & 1.57 & 1.65 & 1.71 & 4.37 & 3.02 & 3.88 & 4.78 & 22.69 \\
\hline 24 & 6.00 & 06.99 & 08.58 & 19.86 & 1.16 & 1.21 & 1.27 & 4.81 & 4.71 & 5.83 & 7.38 & 23.82 \\
\hline 25 & 5.00 & 06.40 & 08.00 & 23.59 & 0.94 & 0.98 & 1.03 & 6.60 & 4.86 & 5.73 & 6.61 & 21.64 \\
\hline 26 & 7.28 & 10.59 & 14.50 & 34.42 & 0.80 & 0.83 & 0.88 & 5.75 & 8.23 & 12.92 & 18.03 & 38.05 \\
\hline 27 & 8.00 & 10.71 & 12.20 & 21.93 & 0.79 & 0.80 & 0.81 & 1.64 & 10.14 & 13.29 & 15.02 & 20.53 \\
\hline
\end{tabular}

Note. SSA: soluble solids content ( ${ }^{\circ}$ Brix), CAA: content of cashew apple in $\%$ of citric acid and ratio: SSA/ CAA. 
Table 5. Average (Ave), minimum (Min) and maximum (Max) values; and coefficient of variation (CV) referring to the chemical characteristics of cashew apple of bushy cashew access

\begin{tabular}{|c|c|c|c|c|c|c|c|c|}
\hline \multirow{2}{*}{ Access } & \multicolumn{4}{|c|}{ VITC } & \multicolumn{4}{|c|}{ CRT } \\
\hline & Min & Ave & Max & $\mathrm{CV}$ & Min & Ave & Max & $\mathrm{CV}$ \\
\hline 1 & 219.93 & 224.46 & 228.99 & 2.86 & 0.12 & 0.16 & 0.19 & 22.62 \\
\hline 2 & 124.63 & 131.81 & 143.74 & 7.89 & 0.17 & 0.18 & 0.19 & 6.16 \\
\hline 3 & 269.19 & 279.64 & 285.96 & 3.26 & 0.15 & 0.20 & 0.22 & 19.81 \\
\hline 4 & 106.28 & 109.23 & 115.10 & 4.65 & 0.21 & 0.25 & 0.30 & 18.92 \\
\hline 5 & 94.08 & 99.28 & 104.54 & 5.27 & 0.14 & 0.29 & 0.56 & 79.85 \\
\hline 6 & 86.28 & 97.93 & 104.64 & 10.34 & 0.30 & 0.33 & 0.37 & 11.20 \\
\hline 7 & 156.52 & 163.57 & 172.28 & 4.89 & 0.20 & 0.28 & 0.27 & 17.30 \\
\hline 8 & 119.54 & 126.37 & 134.11 & 5.80 & 0.27 & 0.30 & 0.33 & 10.01 \\
\hline 9 & 86.49 & 92.91 & 100.52 & 7.63 & 0.22 & 0.27 & 0.34 & 23.50 \\
\hline 10 & 123.83 & 125.04 & 127.39 & 1.63 & 0.08 & 0.10 & 0.12 & 21.14 \\
\hline 11 & 72.13 & 74.09 & 77.31 & 3.80 & 0.15 & 0.21 & 0.26 & 28.03 \\
\hline 12 & 94.44 & 102.80 & 113.49 & 9.47 & 0.21 & 0.24 & 0.26 & 11.88 \\
\hline 14 & 144.02 & 147.27 & 151.17 & 2.46 & 0.22 & 0.23 & 0.25 & 7.84 \\
\hline 15 & 161.78 & 183.72 & 196.07 & 10.37 & 0.17 & 0.18 & 0.19 & 7.41 \\
\hline 16 & 142.26 & 170.58 & 188.44 & 14.54 & 0.19 & 0.30 & 0.37 & 31.85 \\
\hline 17 & 198.88 & 204.51 & 210.83 & 2.93 & 0.09 & 0.16 & 0.22 & 41.14 \\
\hline 19 & 136.03 & 139.71 & 144.72 & 3.22 & 0.10 & 0.15 & 0.20 & 33.56 \\
\hline 20 & 83.88 & 87.53 & 91.47 & 4.34 & 0.24 & 0.28 & 0.32 & 14.14 \\
\hline 21 & 101.42 & 104.52 & 107.49 & 2.91 & 0.17 & 0.20 & 0.23 & 14.66 \\
\hline 22 & 129.72 & 141.09 & 154.74 & 8.97 & 0.15 & 0.18 & 0.21 & 16.22 \\
\hline 23 & 126.82 & 147.63 & 177.87 & 18.15 & 0.17 & 0.19 & 0.21 & 12.38 \\
\hline 24 & 145.58 & 151.83 & 157.53 & 3.95 & 0.21 & 0.27 & 0.31 & 19.07 \\
\hline 25 & 101.91 & 104.06 & 106.21 & 2.92 & 0.17 & 0.27 & 0.37 & 53.51 \\
\hline 26 & 116.96 & 122.43 & 127.59 & 4.35 & 0.48 & 0.48 & 0.48 & 0.49 \\
\hline 27 & 113.19 & 130.72 & 152.78 & 15.44 & 0.10 & 0.14 & 0.17 & 26.37 \\
\hline
\end{tabular}

Note. VITC: vitamin C of cashew apple in $\mathrm{mg} / 100 \mathrm{~g}$ of pulp and CRT: carotenoids total (\%).

\subsection{Physical Traits of Cashew Nut}

Larger cashew nuts are desirable for cashew industry. The average LEN varied from 11.99 to $22.70 \mathrm{~mm}$ (Table 6). For WIN a variation of 11.51 to $22.63 \mathrm{~mm}$ was observed among accessions means. For the THN the variation among evaluated accesses was from 09.35 to $13.69 \mathrm{~mm}$. The mean values of WEN among towns of partners ranged from 0.63 to $3.57 \mathrm{~g}$. For all cashew nut characteristics, access 1 presented higher values, that is, it produces cashew nut with larger dimensions when compared to other accessions. 
Table 6. Average (Ave), minimum (Min) and maximum (Max) values; and coefficient of variation (CV) referring to the physical characteristics of cashew nut of bushy cashew access

\begin{tabular}{|c|c|c|c|c|c|c|c|c|c|c|c|c|c|c|c|c|}
\hline \multirow{2}{*}{ Access } & \multicolumn{4}{|c|}{ LEN } & \multicolumn{4}{|c|}{ WIN } & \multicolumn{4}{|c|}{ THN } & \multicolumn{4}{|c|}{ WEN } \\
\hline & Min & Ave & Max & $\mathrm{CV}$ & Min & Ave & Max & $\mathrm{CV}$ & Min & Ave & Max & $\mathrm{CV}$ & Min & Ave & Max & $\mathrm{CV}$ \\
\hline 1 & 18.00 & 22.70 & 28.29 & 13.24 & 19.35 & 22.63 & 26.15 & 10.17 & 10.97 & 13.69 & 15.88 & 12.04 & 1.45 & 3.57 & 5.71 & 40.12 \\
\hline 2 & 12.94 & 16.14 & 17.89 & 7.55 & 13.75 & 15.34 & 16.57 & 4.64 & 10.54 & 11.77 & 13.09 & 6.23 & 1.13 & 1.47 & 1.83 & 14.26 \\
\hline 3 & 15.74 & 16.78 & 17.95 & 3.36 & 13.56 & 14.42 & 15.68 & 3.67 & 9.77 & 10.47 & 11.09 & 3.65 & 1.02 & 1.18 & 1.35 & 9.28 \\
\hline 4 & 12.31 & 14.20 & 15.49 & 6.01 & 12.18 & 13.82 & 15.22 & 8.03 & 10.32 & 11.13 & 12.12 & 4.35 & 0.88 & 1.30 & 2.67 & 32.06 \\
\hline 5 & 14.06 & 16.61 & 18.42 & 7.67 & 12.61 & 16.50 & 17.54 & 9.72 & 9.22 & 12.93 & 13.96 & 11.85 & 0.51 & 1.84 & 2.19 & 30.01 \\
\hline 6 & 12.13 & 13.58 & 14.65 & 5.55 & 13.42 & 14.33 & 15.44 & 4.56 & 10.13 & 11.11 & 11.76 & 4.79 & 0.83 & 1.08 & 1.21 & 12.68 \\
\hline 7 & 13.58 & 14.96 & 16.64 & 5.46 & 13.00 & 13.95 & 15.36 & 4.61 & 9.63 & 10.57 & 11.35 & 4.17 & 0.98 & 1.18 & 1.38 & 9.02 \\
\hline 8 & 12.17 & 13.67 & 14.97 & 5.82 & 14.10 & 14.85 & 15.90 & 3.82 & 11.83 & 12.43 & 13.36 & 3.85 & 1.13 & 1.33 & 1.61 & 11.97 \\
\hline 9 & 13.88 & 15.44 & 16.67 & 6.47 & 15.53 & 15.93 & 16.61 & 2.53 & 12.04 & 12.33 & 12.82 & 2.38 & 1.31 & 1.55 & 1.78 & 11.76 \\
\hline 10 & 13.73 & 15.48 & 17.36 & 7.37 & 11.50 & 13.82 & 15.49 & 8.81 & 8.10 & 10.69 & 11.89 & 8.11 & 0.87 & 1.20 & 1.72 & 18.41 \\
\hline 11 & 14.56 & 15.57 & 16.75 & 4.46 & 11.88 & 13.95 & 15.16 & 6.47 & 10.46 & 11.31 & 12.39 & 5.78 & 0.85 & 1.05 & 1.43 & 19.00 \\
\hline 12 & 13.66 & 15.63 & 17.58 & 7.94 & 12.96 & 14.05 & 15.26 & 4.21 & 11.01 & 12.12 & 13.84 & 8.14 & 1.20 & 1.49 & 2.03 & 18.26 \\
\hline 13 & 10.19 & 11.99 & 14.06 & 9.67 & 10.42 & 11.51 & 13.03 & 7.79 & 7.92 & 9.35 & 10.83 & 13.03 & 0.31 & 0.63 & 1.02 & 41.21 \\
\hline 14 & 10.88 & 13.27 & 21.65 & 19.95 & 9.94 & 12.54 & 18.55 & 16.46 & 9.50 & 11.38 & 13.99 & 10.56 & 0.72 & 1.05 & 2.47 & 40.83 \\
\hline 15 & 16.17 & 17.60 & 18.84 & 4.78 & 10.08 & 15.22 & 16.98 & 12.26 & 11.04 & 12.47 & 15.55 & 8.53 & 1.47 & 1.78 & 2.22 & 13.09 \\
\hline 16 & 15.01 & 16.12 & 16.53 & 3.88 & 15.97 & 16.69 & 17.18 & 3.10 & 11.83 & 12.13 & 12.42 & 1.86 & 1.55 & 1.66 & 1.72 & 3.99 \\
\hline 17 & 14.10 & 15.43 & 18.01 & 7.24 & 12.15 & 13.28 & 14.83 & 5.45 & 9.99 & 10.64 & 11.35 & 3.84 & 0.89 & 1.31 & 3.88 & 59.48 \\
\hline 18 & 13.09 & 14.46 & 15.77 & 7.40 & 12.17 & 13.57 & 14.23 & 6.19 & 8.86 & 10.14 & 10.72 & 6.44 & 0.66 & 0.93 & 1.04 & 15.43 \\
\hline 19 & 13.91 & 15.47 & 16.81 & 5.48 & 13.72 & 14.72 & 18.40 & 8.43 & 11.43 & 12.18 & 12.96 & 3.83 & 1.03 & 1.37 & 1.82 & 14.92 \\
\hline 20 & 13.13 & 14.02 & 14.78 & 4.91 & 13.08 & 13.94 & 14.71 & 4.37 & 10.02 & 10.57 & 11.14 & 4.64 & 0.65 & 0.97 & 1.14 & 19.79 \\
\hline 21 & 16.61 & 17.06 & 18.29 & 4.07 & 1.88 & 11.74 & 14.81 & 47.17 & 10.02 & 11.11 & 12.43 & 9.20 & 0.85 & 1.40 & 1.76 & 28.91 \\
\hline 22 & 13.39 & 15.15 & 17.84 & 9.42 & 10.70 & 12.54 & 16.83 & 14.99 & 9.83 & 10.83 & 12.50 & 7.36 & 0.81 & 1.16 & 1.70 & 21.74 \\
\hline 23 & 15.56 & 17.32 & 18.25 & 4.94 & 13.24 & 15.31 & 16.17 & 5.05 & 11.78 & 12.79 & 13.39 & 4.33 & 1.11 & 2.75 & 16.98 & 155.83 \\
\hline 24 & 12.78 & 15.19 & 17.74 & 10.11 & 10.88 & 14.18 & 19.93 & 15.34 & 9.46 & 11.48 & 17.40 & 16.85 & 0.78 & 1.15 & 1.51 & 18.02 \\
\hline 25 & 11.48 & 12.25 & 13.46 & 6.56 & 13.54 & 14.13 & 14.78 & 3.65 & 9.37 & 9.72 & 10.06 & 3.27 & 0.59 & 0.73 & 0.84 & 13.82 \\
\hline 26 & 13.16 & 14.69 & 15.63 & 7.10 & 14.29 & 14.88 & 15.76 & 3.28 & 11.66 & 12.10 & 12.51 & 2.37 & 1.15 & 1.29 & 1.46 & 8.26 \\
\hline 27 & 15.46 & 16.75 & 18.38 & 5.17 & 12.94 & 15.88 & 17.16 & 6.22 & 10.29 & 11.35 & 12.93 & 5.45 & 1.19 & 1.88 & 7.88 & 89.33 \\
\hline
\end{tabular}

Note. LEN: cashew nut length (mm). WIN: cashew nut width (mm). THN: cashew nut thickness (mm) and WEN: cashew nut weight $(\mathrm{g})$.

\subsection{Correlation Between the Traits Evaluated}

The evaluation of the fruiting variables of bushy cashew showed that there were significant correlations (Table 7). The highest correlations (above 0.8 ) were observed among the variables: WEA $\times$ LEA (0.887); DAA $\times$ BDA (0.853); WEA $\times$ DAA $(0.863) ;$ WEN $\times$ LEN $(0.882) ;$ WEN $\times$ WIN $(0.827)$, both are considered strong according to the classification of correlation intensity, which according to Guerra and Liveira (1999) for $p<0.01$ is considered very strong ( $\mathrm{r} \pm 0(\mathrm{R} \pm 0.31$ to \pm 0.50$)$, mean ( $\mathrm{r} \pm 0.51$ to \pm 0.70$)$, and weak ( $\mathrm{r} \pm 0.31$ to \pm 0.50$)$. VITC, a desirable trait for cashew, had a negative correlation with hEA (0.4) and hPA (0.461), that is to say, hPA (hue angle of the cashew pulp), the higher the hue angle value, the lower the VITC. 
Table 7. Correlation matrix between the physical and chemical variables of the cashew apple and physical characteristics of the cashew nut of bushy cashew

\begin{tabular}{|c|c|c|c|c|c|c|c|c|c|c|c|c|c|c|c|c|}
\hline & LEA & BDA & DAA & WEA & LEN & WIN & THN & WEN & SSA & CAA & ratio & VITC & CRT & CEA & hEA & CPA \\
\hline$\overline{\mathrm{BDA}}$ & $0.498^{* *}$ & & & & & & & & & & & & & & & \\
\hline DAA & $0.638^{\text {ns }}$ & $0.853^{* *}$ & & & & & & & & & & & & & & \\
\hline WEA & $0.887^{* *}$ & $0.753^{* *}$ & $0.863^{* *}$ & & & & & & & & & & & & & \\
\hline LEN & $0.289^{\text {ns }}$ & $0.269^{\mathrm{ns}}$ & $0.295^{* *}$ & 0.298 & & & & & & & & & & & & \\
\hline WIN & $0.161^{\text {ns }}$ & $0.276^{\mathrm{ns}}$ & $0.394^{*}$ & $0.223^{\mathrm{ns}}$ & $0.754^{* *}$ & & & & & & & & & & & \\
\hline THN & $0.295^{\text {ns }}$ & $0.531^{* *}$ & $0.538^{* *}$ & $0.446^{*}$ & $0.669^{* *}$ & $0.724^{* *}$ & & & & & & & & & & \\
\hline WEN & $0.273^{\mathrm{ns}}$ & $0.375^{\text {ns }}$ & $0.425^{*}$ & $0.347^{\mathrm{ns}}$ & $0.882^{* *}$ & $0.827^{* *}$ & $0.794^{* *}$ & & & & & & & & & \\
\hline SSA & $-0.182^{\mathrm{ns}}$ & $-0.486^{* *}$ & $-0.323^{\text {ns }}$ & $-0.305^{\text {ns }}$ & $0.133^{\text {ns }}$ & $0.049^{\text {ns }}$ & $-0.063^{\text {ns }}$ & $0.053^{\text {ns }}$ & & & & & & & & \\
\hline CAA & $-0.305^{\text {ns }}$ & $-0.031^{\mathrm{ns}}$ & $-0.051^{\mathrm{ns}}$ & $-0.152^{\mathrm{ns}}$ & $0.097^{\text {ns }}$ & $0.063^{\mathrm{ns}}$ & $0.123^{\text {ns }}$ & $0.251^{\mathrm{ns}}$ & $0.099^{\text {ns }}$ & & & & & & & \\
\hline ratio & $0.172^{\mathrm{ns}}$ & $-0.220^{\text {ns }}$ & $-0.072^{\mathrm{ns}}$ & $-0.011^{\text {ns }}$ & $0.088^{\mathrm{ns}}$ & $0.047^{\mathrm{ns}}$ & $0.001^{\text {ns }}$ & $-0.021^{\mathrm{ns}}$ & $0.655^{* *}$ & $-0.619^{* * *}$ & & & & & & \\
\hline VITC & $-0.143^{\mathrm{ns}}$ & $-0.212^{\mathrm{ns}}$ & $-0.096^{\mathrm{ns}}$ & $-0.174^{\mathrm{ns}}$ & $0.441^{*}$ & $0.286^{\text {ns }}$ & $0.059^{\text {ns }}$ & $0.327^{\mathrm{ns}}$ & $0.506^{* *}$ & $0.335^{\mathrm{ns}}$ & $0.200^{\text {ns }}$ & & & & & \\
\hline CRT & $0.126^{\mathrm{ns}}$ & $0.044^{\mathrm{ns}}$ & $0.180^{\text {ns }}$ & $0.190^{\text {ns }}$ & $-0.474^{*}$ & $-0.114^{\mathrm{ns}}$ & $-0.047^{\mathrm{ns}}$ & $-0.314^{\mathrm{ns}}$ & $-0.119^{\mathrm{ns}}$ & $-0.077^{\mathrm{ns}}$ & $-0.017^{\mathrm{ns}}$ & $-0.269^{\text {ns }}$ & & & & \\
\hline CEA & $0.007^{\mathrm{ns}}$ & $0.167^{\mathrm{ns}}$ & $0.030^{\mathrm{ns}}$ & $0.037^{\mathrm{ns}}$ & $0.160^{\text {ns }}$ & $-0.054^{\mathrm{ns}}$ & $0.042^{\mathrm{ns}}$ & $0.035^{\mathrm{ns}}$ & $0.131^{\mathrm{ns}}$ & $-0.088^{\mathrm{ns}}$ & $0.108^{\mathrm{ns}}$ & $0.156^{\mathrm{ns}}$ & $-0.277^{\mathrm{ns}}$ & & & \\
\hline $\mathrm{hEA}$ & $0.101^{\mathrm{ns}}$ & $0.198^{\mathrm{ns}}$ & $0.086^{\mathrm{ns}}$ & $0.124^{\text {ns }}$ & $-0.488^{* *}$ & $-0.606^{* *}$ & $-0.263^{\mathrm{ns}}$ & $-0.535^{* *}$ & $-0.194^{\mathrm{ns}}$ & $-0.440^{*}$ & $0.160^{\text {ns }}$ & $-0.420^{*}$ & $0.006^{\mathrm{ns}}$ & $0.258^{\mathrm{ns}}$ & & \\
\hline CPA & $-0.490^{* *}$ & $-0.304^{\mathrm{ns}}$ & $-0.355^{\mathrm{ns}}$ & $-0.473^{*}$ & $-0.438^{*}$ & $-0.441^{*}$ & $-0.305^{\mathrm{ns}}$ & $-0.516^{* *}$ & $0.196^{\mathrm{ns}}$ & $-0.169^{\mathrm{ns}}$ & $0.269^{\text {ns }}$ & $0.080^{\text {ns }}$ & $0.197^{\mathrm{ns}}$ & $0.282^{\text {ns }}$ & $0.398^{*}$ & \\
\hline $\mathrm{hPA}$ & $0.121^{\mathrm{ns}}$ & $0.200^{\text {ns }}$ & $0.204^{\mathrm{ns}}$ & $0.232^{\mathrm{ns}}$ & $-0.598^{* *}$ & $-0.644^{* *}$ & $-0.319^{\text {ns }}$ & $-0.583^{* *}$ & $-0.324^{\mathrm{ns}}$ & $-0.180^{\text {ns }}$ & $-0.109^{\text {ns }}$ & $-0.461^{*}$ & $0.109^{\text {ns }}$ & $-0.090^{\text {ns }}$ & $0.756^{* *}$ & $0.116^{\mathrm{n}}$ \\
\hline
\end{tabular}

Note. LEA: cashew apple length $(\mathrm{mm})$, BDA: cashew apple base diameter $(\mathrm{mm})$, DAA: cashew apple apex diameter $(\mathrm{mm})$ and WEA: cashew apple weight $(\mathrm{g})$, LEN: cashew nut length $(\mathrm{mm})$, WIN: cashew nut width (mm), THN: cashew nut thickness (mm), WEN: cashew nut weight (g), SSA: soluble solids content ( ${ }^{\circ}$ Brix), CAA: content of cashew apple in \% of citric acid and ratio: SSA/CAA, VITC: vitamin C of cashew apple in $\mathrm{mg} / 100 \mathrm{~g}$ of pulp and CRT: carotenoids total (\%), CEA: coordinated $\mathrm{C}^{*}$ of cashew apple epidermis, hEA: hue angle $\left(\mathrm{h}^{\circ}\right)$ of cashew apple epidermis, CPA: coordinated $\mathrm{C}^{*}$ of cashew apple pulp and hPA: hue angle $\left(\mathrm{h}^{\circ}\right)$ of cashew apple pulp.

**, *: significant at 1 and $5 \%$ probability by the t-test. ${ }^{\text {ns }}$ not significant.

\section{Discussion}

Santos and Santos Júnior (2015) found average LEA of $17.2 \mathrm{~mm}$ on cashew apple in the cerrado of Minas Gerais state, Gomes et al. (2013) working with cashew apple obtained in the area of Embrapa Mid-North in Teresina, in Piauí state, observed variation from 14.89 to $35.42 \mathrm{~mm}$. Studing cashew apple also derive from the state of Piaui, Rocha, Figueiredo, Araújo, and Moreira-Araújo (2013), they found average values of LEA of $34.2 \mathrm{~mm}$ and WEA of $7.9 \mathrm{~g}$, in the present research it was found mean values superior to those found by Santos and Santos Junior (2015), in which the average WEA was $3.26 \mathrm{~g}$, indicating that the cashew apple of this work has greater weight and, possibly, larger size. Gomes et al. (2013) was also observed wide variation, with average values between 5.11 and $35.41 \mathrm{~g}$. It should be noted that the weight values found by that author was superior to those found in this study, even the close values for length, it is assumed, then, that the present work detected smaller diameter. The higher values found by Gomes et al. (2013) can also be explained due to the fact of cashew apple of the present work is originated from a germplasm bank, where the plants were not in their natural habitat, and perhaps, have not adapted to the environment. According Gonzaga Neto, Mattuz, and Santos (1999), the greater the fruit (cashew apple), easier and faster their harvest, requiring less labor and, consequently, reducing production costs, in addition to being more attractive for in nature consumption.

Cashew nuts with larger dimensions are more desirable for cashew industry. The WEN average values, in the majority of accesses, are superior to those found by Santos and Santos Junior (2015). They found an average value of $1.00 \mathrm{~g}$ and a mean value of $14.00 \mathrm{~mm}$ for LEN in cashew nut in the cerrado of Minas Gerais state.There was a variation of 0.63 to $4.16 \mathrm{~g}$ and an mean value of $2.51 \mathrm{~g}$ at Gomes et al. (2013) research, in wich data were obtained from fruits of natural occurrence at Embrapa Mid-North in Teresina, at Piauí state, wich may have favored the slight superiority found in relation to the present work. The adaptability of plants can influence, also, in the dimensions of the cashew nut, since the cashew nut of this work were obtained from a germplasm bank, where the plants were in an environment other than the original one. The genetics of the accesses and the edaphoclimatic conditions to which they are subject are also factors that influence the quality of cashew nut.

The SSA are compounds that are soluble in water and indicate the amount of sugars in fruit, whereas other compounds, although in small proportions also take part, as, for example, acids, vitamins, amino acids and some of the pectins (Kluge, Nachtigal, Fachinelo, \& Bilhalva, 2002; M. I. F. Chitarra \& A. B. Chitarra, 2005). The preference of Brazilian consumers is for fruits with low to medium acidity and with high content of soluble solids (Trevisan, Piana, Treptow, Gonçalves, \& Antunes, 2010). 
The variation observed for the percentage of CAA values corroborate to those found by Gomes et al. (2013), who observed variation of 0.11 to $1.27 \%$ and $0.77 \%$. According with Brazilian laws, the common cashew pulp (Anacardium ocidentale) must contain at least $0.30 \%$ of citric acid (Brasil, 2000). Cashew apple with high amounts of acid are desirable for industry, since the acid acts as a preservative, requiring less adding artificial preservatives.

The ratio is a quality parameter for fruit acceptance, being an important indicator of flavor (Almeida \& Durigan, 2006), because it is related the sugars and fruit acids. It is assumed that higher ratio values indicate higher fruits sweetness (Silva, Figueiredo, \& Lima, 2016), and it is an important variable to be considered in the selection for those who are destined for in nature consumption.

The carotenoids are important not only as precursors of vitamin A, but also because they have considerable antioxidant activity (Rufino et al., 2010; Veronezi \& Jorge, 2011) associated with a decreased risk of degeneration diseases (Veronezi \& Jorge, 2011). The contents of the studied accesses, confirms its nutritional potential. The need for reliable data about carotenoids in foods is widely recognized in various fields of study. The factors that make this analysis difficult include the large number of carotenoids naturally existing, the qualitative and quantitative variation of these foods, the little amount of pro-vitamin A carotenoids, its varied biopotency and the fact of the carotenoids are extremely unsaturated molecules, providing isomerisation processes, oxidation and degradation during the analysis (Matias et al., 2016). According Silva, Cremasco, Matias, Silva, and Bruckner (2014), the levels of total carotenoids concentration characterizes the yellow coloration of the pulp and in accordance with Faraoni, Ramos, and Stringheta (2008), in the pulp and products produced on the basis of fruit pulp, the color is an important parameter used in quality control of these products. Trevisan et al. (2010), evaluating the preferences of consumers in natura of peach in three cities of Rio Grande do Sul, found that $70 \%$ of consumers prefer peaches with reddish skin, demonstrating the influence of color on consumer choice.

The VITC is a synergistic antioxidant and oxygen remover. It acts directly with oxygen, forming the dehydroacetic ascorbic, eliminating the supply of oxygen available for auto-oxidation reactions (Daiuto, Vieites, \& Carvalho, 2011). Hojo, Durigan, and Hojo (2011) stated that, during senescence, the ascorbic acid content of the fruit is used in oxidative reactions, which are activated by the stress suffered by cellular membranes during this period, which contributes to a reduction in the levels of ascorbic acid. According to the table of food composition (Nepa, 2011), the cashew nuts has $219.3 \mathrm{mg} / 100 \mathrm{~g}$ and the frozen pulp has 119.7 of VITC. In the present study, there was variation among the measured values. A large part of the accessions showed mean values close to those presented at the table of composition and are above the recommended for daily intake brazilian health department, which is $60 \mathrm{mg}$ of vitamin C for adults (Brasil, 2003), indicating that the bushy cashew is an important source of vitamin $\mathrm{C}$.

The high instability of vitamins and pro-vitamins, during the ripening of fruits can result in significant changes in the qualitative and quantitative composition of some nutrients (Matias et al., 2016). According to Silva et al. (2014) during the fruits ripening it may occur nutrient losses depending on the environmental conditions. The decomposition of ascorbic acid has been reported as the most deteriorating reaction that occurs during the ripening process.

The accessions showed to be distinct regarding the superiority in the evaluated variables. Access 5 showed superiority regarding the physical characteristics of cashew apple, whereas for cashew nut size, access 1 presented higher values and differentiated color (red) in the cashew apple epidermis, while access 3 has higher levels of SSA and VITC, the access 23 higher levels of CAA and access 26 higher values of CRT. These results are interesting when it comes to a germplasm bank, since it shows that it is representative of the diversity of the genetic material of the species in the region. It is important to emphasize that for the possibility of implementing an improvement program, it is necessary that several genotypes are included and not only the superior one. Therefore more detailed later studies involving the accesses mentioned in the present work are necessary.

The bushy cashew is not yet domesticated, in this way, studies related to its characterization, cultivation, conservation and processing are of extreme importance due to the large potential for income generation, mainly in the areas of occurrence of the specie.

\section{Conclusion}

Most of the accessions presented high CV for most of the variables, demonstrating a high heterogeneity of the observed values.

Access 1 presented larger dimensions of cashew nuts, demonstrating great potential for industry. 
Cashew apples have high levels of vitamin $\mathrm{C}$ and carotenoids, indicating the nutritional potential of the specie.

Access 3 has higher levels of vitamin C and SSA, which makes it interesting for consumption in natura.

High nutritional values of the species reinforce the need for more valorization and use, as a process of maturing and understanding of preservation; and the possibility of commercial crops for employment and income generation.

\section{References}

Almeida, G. V. B., \& Durigan, J. F. (2006). Relação entre as características químicas e o valor dos pêssegos comercializados pelo sistema veiling frutas Holambra em Paranapanema-SP. Revista Brasileira de Fruticultura, 28, 218-221. https://doi.org/10.1590/S0100-29452006000200014

Almeida Júnior, E. B., Chaves, L. J., \& Soares, T. N. (2014). Genetic characterization of a germplasm collection of cagaiteira, a native species of the cerrado. Bragantia, 73, 246-252. https://doi.org/10.1590/16784499.0075

Avidos, M. F. D., \& Ferreira, L. T. (2000). Frutos dos cerrados: Preservação gera muitos frutos. Biotecnologia Ciencia \& Desenvolvimento, 3(15), 36-41.

Brasil. (2000). Instrução Normativa no. 01, de 7 de Janeiro de 2000. Regulamento técnico geral para fixação dos padrões de identidade e qualidade para polpa de fruta. Regulamento Técnico Sobre Rotulagem Nutricional de Alimentos Embalados. Brasília-DF.

Brasil. (2003). Resolução ANVISA/MS RDC no. 360, de 23 de Dezembro de 2003. Regulamento Técnico Sobre Rotulagem Nutricional de Alimentos Embalados. Brasília-DF.

Bruno, L. R. G. P., Antonio, R. P., de Aquino Assis, J. G., Moreira, J. N., \& Lira, I. C. D. S. A. (2017). Caracterização morfoagronômica de capim buffel do banco ativo de germoplasma de Cenchrus. Revista Caatinga, 30(2), 487-495. https://doi.org/10.1590/1983-21252017v30n224rc

Burle, M. L., \& Oliveira, M. S. P (2010). Manual de Curadores de Germoplasma-Vegetal: Caracterização Morfológica (p. 15). Embrapa Recursos Genéticos e Biotecnologia, Documentos, 312; Embrapa Amazônia Oriental, 378. Brasília, DF: Embrapa Recursos Genéticos e Biotecnologia; Belém, PA: Embrapa Amazônia Oriental.

Chitarra, M. I. F., \& Chitarra, A. B. (2005). Pós-colheita de frutas e hortaliças: Fisiologia e manuseio (2nd ed.). Universidade Federal De Lavras, Lavras, MG.

Cruz, C. D. (2013). GENES-A software package for analysis in experimental statistics and quantitative genetics. Acta Scientiarium, 35(3), 271-276.

Daiuto, E. R., Vieites, R. L., \& Carvalho, L. R. (2011). Avaliação sensorial do guacamole com adição de á-tocoferol e ácido ascórbico conservado pelo frio. Revista Ceres, 58, 140-148. https://doi.org/10.1590/ S0034-737X2011000200002

Faraoni, A. S., Ramos, A. M., \& Stringheta, P. R. (2008). Efeito dos métodos de conservação, tipos de embalagem e tempo de estocagem na coloração de polpa de manga 'Ubá' produzida em sistema orgânico. Revista Ceres, 55, 504-511.

Gomes, S. O., Souza, V. A. B., Costa, M. P. S. D., Silva, C. C. P., Vale, E. M., Souza, M., \& Souza, J. P. B. (2013). Avaliação da qualidade física e química de cajuí (Anacardium spp.) na região Meio-norte: Uma prospecção tecnológica. Revista Geintec, 3, 139-145. https://doi.org/10.7198/S2237-07222013000300013

Gonzaga Neto, L., Mattuz, B. H., \& Santos, C. A. F. (1999). Caracterização agronômica de clones de aceroleira (Malpighia spp.) na região do submédio São Francisco. Revista Brasileira de Fruticultura, 21, 110-115.

Guerra, N. B., \& Liveira, A. V. S. (1999). Correlação entre o perfil sensorial e determinações físicas e químicas do abacaxi cv. Pérola. Revista Brasileira de Fruticultura, 21(1), 32-35.

Hojo, E. T. D., Durigan, J. F., \& Hojo, R. H. (2011). Uso de tratamento hidrotérmico e ácido clorídrico na qualidade de lichia 'Bengal'. Revista Brasileira de Fruticultura, 33, 386-393. https://doi.org/10.1590/ S0100-29452011005000057

Instituto Adolfo Lutz. (2008). Métodos físico-químicos para análise de alimentos (4th ed./1st ed.). São Paulo, SP.

Kluge, R. A., Nachtigal, J. C., Fachinelo, J. C., \& Bilhalva, A. B. (2002). Fisiologia e manejo pós-colheita de frutas de clima temperado (2nd ed.). Livraria e Editora Rural, Campinas, SP. 
Lichtenthaler, H. K. (1987). Chlorophylls and carotenoids: Pigment photosynthetic biomembranes. Methods Enzymol, 148, 362-385. https://doi.org/10.1016/0076-6879(87)48036-1

Lima, V. P. M. S. A. (1988). Cultura do cajueiro no nordeste do Brasil (pp. 15-61). Banco do Nordeste do Brasil, Fortaleza, CE.

Londe, L. N., Ribeiro, E. B., Sousa, C. S., Kerr, W. E., \& Bonetti, A. M. (2010). Divergência genética entre populações de Anacardium humile St. Hill por marcadores AFLP. Circular Técnica Epamig, 105 (pp. 1-4). Epamig.

Matias, R. G. P., Silva, D. F. P., Miranda, P. M. D., Oliveira, J. A. A., Pimentel, L. D., \& Bruckner, C. H. (2016). Relationship between fruit traits and contents of ascorbic acid and carotenoids in peach. Crop Breeding and Applied Biotechnology, 16, 348-354. https://doi.org/10.1590/1984-70332016v16n4n51

Moura, N. F., Chaves, L. J., \& Naves, R. V. (2013). Caracterização física de frutos de pequizeiro (Caryocar brasiliense Camb.) do cerrado. Revista Árvore, 37, 905-912. https://doi.org/10.1590/S0100-67622013000 500013

NEPA (Núcleo de Estudos e Pesquisas em Alimentação). (2011). Tabela brasileira de composição de alimentos (4th ed., p. 37).

Rocha, M. S., Figueiredo, R. W., Araújo, M. A. M., \& Moreira-Araújo, R. S. R. (2013). Caracterização físico-química e atividade antioxidante (in vitro) de frutos do Cerrado Piauiense. Revista Brasileira de Fruticultura, 35, 933-941. https://doi.org/10.1590/S0100-29452013000400003

Rufino, M. S. M., Alves, R. E., Brito, E. S., Pérez-Jiménez, J., Saura-Calixto, F., \& Mancini-Filho, J. (2010). Bioactive compounds and antioxidant capacities of 18 non-traditional tropical fruits from Brazil. Food Chemistry, 121, 996-1002. https://doi.org/10.1016/j.foodchem.2010.01.037

Santos, R. C., \& Santos Júnior, J. E. (2015). Divergência genética por análise multivariada de caracteres fenotípicos de Anacardium humile (St. Hilaire). Revista Ceres, 62, 507-509. https://doi.org/10.1590/0034737 X201562060007

Silva, D. F. P., Siqueira, D. L., Rocha, A., Salomão, L. C. C., Matias, R. G. P., \& Struiving, T. B. (2012). Diversidade genética entre cultivares de mangueiras, baseada em caracteres de qualidade dos frutos. Revista Ceres, 59, 225-232. https://doi.org/10.1590/S0034-737X2012000200011

Silva, D. F. P., Cremasco, J. P. G., Matias, R. G. P., Silva, J. O. C., \& Bruckner, C. H. (2014). Degradação de antioxidantes e sólidos solúveis em polpa de pêssego. Revista Magistra, 26, 1136-1140.

Silva, M. R., Lacerda, D. B. C. L., Santos, G.G., \& Martins, D. M. O. (2008). Caracterização química de frutos nativos do cerrado. Ciência Rural, 38(6), 1790-1793. https://doi.org/10.1590/S0103-84782008000600051

Silva, Q. J., Figueiredo, F. J., \& Lima, V. L. A. G. (2016). Características físicas e químicas de ciriguelas cultivadas na Zona da Mata Norte de Pernambuco. Revista Ceres, 63, 285-290. https://doi.org/10.1590/ 0034-737X201663030002

Silva-Luz, C. L., \& Pirani, J. R. (2010). Anacardiaceae. Lista de espécies da flora do Brasil. Retrieved from http://floradobrasil.jbrj.gov.br/2010/FB015463

Trevisan, R., Piana, C. F. B., Treptow, R. O., Gonçalves, E. D., \& Antunes, L. C. C. (2010). Perfil e preferências do consumidor de pêssego (Prunus persica) em diferentes regiões produtoras no Rio Grande do Sul. Revista Brasileira de Fruticultura, 32, 90-100. https://doi.org/10.1590/S0100-29452010005000011

Veronezi, C. M., \& Jorge, N. (2011). Carotenóides em abóboras. Boletim do Centro de Pesquisa de Processamento de Alimentos, 29, 9-20. https://doi.org/10.5380/cep.v29i1.22734

\section{Copyrights}

Copyright for this article is retained by the author(s), with first publication rights granted to the journal.

This is an open-access article distributed under the terms and conditions of the Creative Commons Attribution license (http://creativecommons.org/licenses/by/4.0/). 